ARTICLE OPEN

\title{
Emerging 2D-ferromagnetism and strong spin-orbit coupling at the surface of valence-fluctuating $\mathrm{EuIr}_{2} \mathrm{Si}_{2}$
}

Susanne Schulz ${ }^{1}$, Ilya A. Nechaev ${ }^{2,3}$, Monika Güttler ${ }^{1}$, Georg Poelchen ${ }^{1}$, Alexander Generalov ${ }^{4}$, Steffen Danzenbächer ${ }^{1}$, Alla Chikina ${ }^{5}$, Silvia Seiro $\mathbb{D}^{6}$, Kristin Kliemt $\mathbb{D}^{7}$, Alexandra Yu. Vyazovskaya ${ }^{8,9}$, Timur K. Kim (iD) ${ }^{10}$, Pavel Dudin (D) $^{10}$, Evgueni V. Chulkov $^{2,3,8,9,11}$, Clemens Laubschat ${ }^{1}$, Eugene E. Krasovskii ${ }^{3,11,12}$, Christoph Geibel ${ }^{10}{ }^{13}$, Cornelius Krellner (iD) ${ }^{7}$ Kurt Kummer ${ }^{14}$ and Denis V. Vyalikh ${ }^{3,11,12}$

The development of materials that are non-magnetic in the bulk but exhibit two-dimensional (2D) magnetism at the surface is at the core of spintronics applications. Here, we present the valence-fluctuating material Eulr ${ }_{2} \mathrm{Si}_{2}$, where in contrast to its nonmagnetic bulk, the Si-terminated surface reveals controllable 2D ferromagnetism. Close to the surface the Eu ions prefer a magnetic divalent configuration and their large $4 \mathrm{f}$ moments order below $48 \mathrm{~K}$. The emerging exchange interaction modifies the spin polarization of the 2D surface electrons originally induced by the strong Rashba effect. The temperature-dependent mixed valence of the bulk allows to tune the energy and momentum size of the projected band gaps to which the $2 \mathrm{D}$ electrons are confined. This gives an additional degree of freedom to handle spin-polarized electrons at the surface. Our findings disclose valence-fluctuating rare-earth based materials as a very promising basis for the development of systems with controllable 2D magnetic properties which is of interest both for fundamental science and applications.

npj Quantum Materials (2019)4:26; https://doi.org/10.1038/s41535-019-0166-z

\section{INTRODUCTION}

Its proximity to the center of the lanthanide period makes elemental europium highly interesting for the elaboration of novel materials with rich and exotic properties that are governed by the $4 f$ electrons and their interactions. ${ }^{1-3}$ In solids the rare earth atoms are usually in the trivalent state which in the case of Eu results in a $4 f^{6}(5 d 6 s)^{3}$ configuration. However, the $4 f^{6}$ shell has a much lower Hund's rule polarization energy than the half-filled $4 f^{7}$ shell. ${ }^{4}$ Therefore, in Eu systems it can be energetically more favorable to move one electron from the valence states to the localized $4 f$ shell resulting in a divalent Eu state with a $4 f^{7}\left(6 s^{2}\right)$ configuration. This is connected with a pronounced change in the magnetic properties: according to Hund's rules, the trivalent Eu configuration has a non-magnetic ground state because of a total angular momentum $\mathrm{J}=0$, while the divalent Eu state has a large, pure spin moment of $7 \mu_{B}$ because of $\mathrm{J}=\mathrm{S}=7 / 2$. This makes divalent europium an outstanding element for designing materials which might be of interest for magnetic devices, in particular at the nanoscale where two-dimensional (2D) electrons may be polarized due to exchange interaction with the ordered $4 f$ moments of magnetically-active Eu atoms.

Because the magnetic divalent and non-magnetic trivalent configuration are close to each other in energy, either a magnetic $4 f^{7}$ or a non-magnetic $4 f^{6}$ state can be stabilized in Eu systems, depending on external parameters like hydrostatic pressure, temperature or internal parameters such as chemical pressure, surrounding ligands, hole or electron doping. ${ }^{1-3,5,6}$ Moreover, manipulation of these parameters can also lead to a valencefluctuating state as has been observed in a number of Eu materials like $\mathrm{EuPd}_{2} \mathrm{Si}_{2}{ }^{7} \mathrm{EuNi}_{2} \mathrm{P}_{2}{ }^{8}$ and $\mathrm{EuIr}_{2} \mathrm{Si}_{2}$. ${ }^{9}$ Such a state is nonmagnetic, or, more precisely, does not possess magnetic order. This balance at the edge between a strongly magnetic and nonmagnetic state makes these phenomena highly relevant and attractive for material design, targeting systems with well predictable and effectively controllable properties. The magnetic, non-magnetic and valence-fluctuating properties of Eu materials are routinely investigated separately, but their combination in one system has not been considered much yet.

Here, we explore Eulr ${ }_{2} \mathrm{Si}_{2}$, which in the bulk reveals a fluctuating valence of the Eu ions continuously changing from about 2.35 at room temperature to about 2.83 at low temperature. ${ }^{9-11}$ Remarkably, in the surface region we discovered very different properties. When Eulr ${ }_{2} \mathrm{Si}_{2}$ is terminated by $\mathrm{Si}$, relaxation of the topmost Si-Ir-Si block tends to create more space for the Eu ions in the fourth layer, and thus stabilizes the magnetic $4 f^{7}$ configuration, which has a slightly larger ionic radius. As a consequence, a magnetically active Eu layer is formed below the Si-Ir-Si silicide trilayer on top of the non-magnetic bulk material. A further

\footnotetext{
${ }^{1}$ Institut für Festkörper- und Materialphysik, Technische Universität Dresden, D-01062 Dresden, Germany; ${ }^{2}$ Centro de Física de Materiales CFM-MPC and Centro Mixto CSIC-UPV/ EHU, 20018 Donostia/San Sebastián, Basque Country, Spain; ${ }^{3}$ Donostia International Physics Center (DIPC), 20018 Donostia/San Sebastián, Basque Country, Spain; ${ }^{4}$ MAX IV Laboratory, Lund University, PO Box 118, 22100 Lund, Sweden; ${ }^{5}$ Swiss Light Source, Paul Scherrer Institute, CH-5232 Villigen-PSI, Switzerland; ${ }^{6}$ IFW Dresden, Helmholtzstr. 20, D01069 Dresden, Germany; ${ }^{7}$ Kristall- und Materiallabor, Physikalisches Institut, Goethe-Universität Frankfurt, Max-von-Laue Strasse 1, D-60438 Frankfurt am Main, Germany; ${ }^{8}$ Tomsk State University, Lenina Av. 36, 634050 Tomsk, Russia; ${ }^{9}$ Saint Petersburg State University, Saint Petersburg 198504, Russia; ${ }^{10}$ Diamond Light Source, Harwell Campus, Didcot OX11 ODE, UK; ${ }^{11}$ Departamento de Física de Materiales UPV/EHU, 20080 Donostia/San Sebastiań, Basque Country, Spain; ${ }^{12}$ IKERBASQUE, Basque Foundation for Science, 48013 Bilbao,

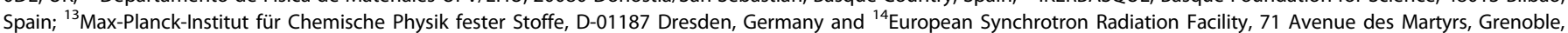
France

Correspondence: Denis V. Vyalikh (Denis.Vyalikh@dipc.org)
}

Received: 8 January 2019 Accepted: 13 May 2019

Published online: 03 June 2019 
interesting feature, which makes the system even more peculiar, originates from the spin-orbit splitting of the Si-terminated surface states-a strong Rashba effect due to the presence of the heavy Ir atoms in the silicide trilayer. Its combination with the exchange magnetic interaction of surface-state electrons with the divalent Eu layer underneath the topmost Si-Ir-Si trilayer opens perspectives to explore how itinerant 2D electrons within the outermost layers are affected and how their properties can be controlled by means of spin-orbit and exchange magnetic interaction at the surface of a non-magnetic Eu system.

\section{RESULTS AND DISCUSSION}

Photoemission insight into surface and bulk properties

We consider a Si-terminated crystal of Eulr ${ }_{2} \mathrm{Si}_{2}$ and focus on the properties of the Si-Ir-Si-Eu (SISE) block forming the surface. In order to trace the anticipated temperature-dependent valence change of Eu in Eulr ${ }_{2} \mathrm{Si}_{2}{ }^{9,11}$ by photoemission (PE), we took survey spectra using photon energies sensitive to the emission of Eu $4 f$ states. Making use of the $4 d \rightarrow 4 f$ Fano resonance we established that the $\mathrm{Eu}^{2+}$ and $\mathrm{Eu}^{3+} \mathrm{PE}$ signals are resonantly enhanced at photon energies of $\sim 141.5$ and $\sim 145 \mathrm{eV}$, respectively. In Fig. 1 we show angle-integrated PE spectra, which were taken at 200 and $7 \mathrm{~K}$ with photons of $145 \mathrm{eV}$ and normalized to the maximum of the $\mathrm{Eu}^{2+}$ emission. It can immediately be seen that in contrast to the general expectation both PE spectra are characterized by a pronounced $\mathrm{Eu}^{2+}$ signal close to the Fermi energy $\mathrm{E}_{F}$. This is curious, as this holds true also for the PE spectrum taken at $7 \mathrm{~K}$ when the bulk of Eulr ${ }_{2} \mathrm{Si}_{2}$ is expected to be almost trivalent, and thus the $\mathrm{Eu}^{3+}$ signal at binding energies (BEs) above $6 \mathrm{eV}$ should dominate over the $\mathrm{Eu}^{2+}$ emission. However, it can be clearly seen that the trivalent signal is only moderately enhanced and remains much weaker than the $\mathrm{Eu}^{2+}$ signal even at low temperature. This remarkable observation suggests that in the subsurface layer the Eu valence is different from that in the bulk. It is thus reasonable to propose that it might be stable divalent, i.e. magnetically active.

Let us now discuss the given spectra in more detail. In mixedvalent $\mathrm{Eu}$ compounds the $4 f^{6}$ final state of $\mathrm{Eu}^{2+}$ is almost degenerate with the ground state and appears close to $\mathrm{E}_{F}$ while the $4 f^{5}$ final state of $\mathrm{Eu}^{3+}$ is shifted to higher BEs. The separation between these two final-state multiplets by the Coulomb

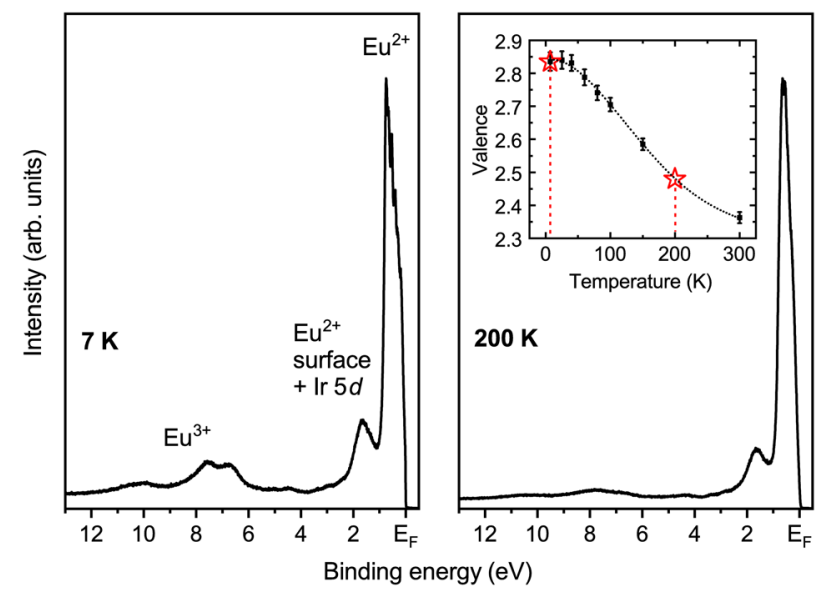

Fig. 1 The $4 f$-derived electron emission of $\mathrm{Eulr}_{2} \mathrm{Si}_{2}$ probed at different temperatures. Angle-integrated energy-distribution curves taken at the $4 d \rightarrow 4 f$ resonance sensitive to the $\mathrm{Eu}^{3+}$ configuration at a photon energy of $145 \mathrm{eV}$ from a Si-terminated surface of the mixed-valent compound $\mathrm{Eulr}_{2} \mathrm{Si}_{2}$. The temperature dependence of the valence as obtained by XAS is given as inset. ${ }^{11}$ Red stars mark the valence of Eulr $\mathrm{Si}_{2}$ in the bulk at $7 \mathrm{~K}(v=2.83)$ and $200 \mathrm{~K}(v=2.5)$ at which the PE spectra were acquired repulsion $\mathrm{U}_{f f}$ is about $6 \mathrm{eV}$. The small peak seen at $1.6 \mathrm{eV} \mathrm{BE}$ is the known surface-core-level-shift signal which stems from divalent Eu atoms at the surface. Its low intensity suggests that the probed crystallite is predominantly terminated by $\mathrm{Si}$ and fractions with Eu-terminated areas are rather negligible. ${ }^{12}$ Note that a finite contribution from Ir $5 d$ states cannot be excluded, since the respective Cooper minimum is not very pronounced. The inset of Fig. 1 shows the temperature dependence of the Eu valence derived from bulk-sensitive X-ray absorption spectroscopy (XAS) measurements. ${ }^{11}$ They imply that the Eu valence $v$ is equal to 2.5 at $200 \mathrm{~K}$ and gradually increases up to about 2.83 at $7 \mathrm{~K}$ as indicated by red stars. Undoubtedly, the bulk-sensitive XAS results are in contradiction with our surface-sensitive PE measurements. On the basis of the performed analysis (see also Supplementary Note 1), we propose that for the Si-terminated surface of Eulr ${ }_{2} \mathrm{Si}_{2}$ the first Eu layer hidden by the topmost Si-Ir-Si trilayer is divalent. Our proposition is also supported by recent photoemission studies of other Eu-based compounds. For example, a divalent subsurface was reported for the isostructural, mixed-valent materials EuPd ${ }_{2} \mathrm{Si}_{2}{ }^{13,14}$ and $\mathrm{EuNi}_{2}\left(\mathrm{Si}_{0.2} \mathrm{Ge}_{0.8}\right)_{2} .^{15}$

The proposed divalence of $\mathrm{Eu}$ in the subsurface of nonmagnetic Eulr $\mathrm{Si}_{2}$ is remarkable and raises the question if the Eu $4 f$ moments in the fourth layer can be ferromagnetically (FM) ordered and if $2 \mathrm{D}$ magnetism can evolve in the surface region of mixed-valent Eulr $_{2} \mathrm{Si}_{2}$. A direct and simple way to examine whether the $\mathrm{Eu} 4 \mathrm{f}$ moments order is to investigate the surface electronic state associated with the Si-terminated surface. This state is located around the $\bar{M}$-point of the surface Brillouin zone (SBZ) within a large gap of the surface-projected bulk bands. In the following we will therefore refer to the gap as the $\bar{M}$-gap and to the surface state as the $\bar{M}$ surface state. The latter is an intrinsic feature of the Si-terminated surface of isostructural $R E R h_{2} \mathrm{Si}_{2}$ compounds (RE $=\mathrm{Eu}, \mathrm{Gd}, \mathrm{Ho}, \mathrm{Dy}, \mathrm{Tb}$ ). ${ }^{16-19}$ The respective surfacestate electrons are largely localized in the Si-Rh-Si-RE block building up the four topmost layers of the Si-terminated crystal. When the system moves into the magnetically ordered regime, the $\bar{M}$ surface state experiences a strong exchange-induced splitting due to coupling with the $4 f$ moments in the fourth layer below the Si surface. ${ }^{17-19}$ Note that in contrast to Eulr ${ }_{2} \mathrm{Si}_{2}$ the mentioned $\mathrm{RERh}_{2} \mathrm{Si}_{2}$ compounds are bulk antiferromagnets (AFM) in which rare-earth FM planes are stacked antiferromagnetically along the $c$ axis. From the band dispersion of the $\bar{M}$ surface state conclusions on the in or out of plane alignment of the $4 \mathrm{fmoments}$ can be drawn. ${ }^{19}$ In this sense the $\bar{M}$ surface state acts as an indicator for the magnetic properties of the surface Si-Rh-Si-RE block. By exploiting this observation, we use angle-resolved photoelectron spectroscopy (ARPES) to explore the temperature dependence of the electronic structure of Si-terminated $\mathrm{EuIr}_{2} \mathrm{Si}_{2}$ and focus particularly on the spectral pattern reflecting the $\overline{\mathrm{M}}$ surface state.

Having proposed a divalent Eu configuration in the fourth layer the reasonable question arises: Can we visualize the expected mixed-valent properties of $\mathrm{Eu}$ in the bulk of $\mathrm{EuIr}_{2} \mathrm{Si}_{2}$ with surfacesensitive ARPES? To answer this question we will now consider the ARPES-derived Fermi surface (FS) from Si-terminated Eulr ${ }_{2} \mathrm{Si}_{2}$ taken at $200 \mathrm{~K}(v=2.5)$ and $7 \mathrm{~K}(v=2.83)$ shown in Fig. 2 . Let us first pay attention to the large, pronounced feature centered at the $\bar{\Gamma}$-point. According to the extended knowledge on surface and bulk ARPES features in $\mathrm{RET}_{2} \mathrm{Si}_{2}(\mathrm{~T}=\mathrm{Rh}, \mathrm{Ir})$ systems, this feature has been shown to correspond to one part of the bulk Fermi surface. Owing to its shape, it is usually referred to as the Doughnut and marked as $\mathcal{D}$ in Fig. 2. ${ }^{16,20,21}$ A direct comparison of the Doughnut on the left and right side of Fig. 2 shows that the temperature change leads to a significant resizing of the latter. At $200 \mathrm{~K}$, when the bulk valence of Eu is 2.5 , we observe a large Doughnut with its necks extending almost up to the $\bar{X}$-points of the SBZ, while at $7 \mathrm{~K}(v=2.83)$ they are dragged inwards resulting in a notably smaller size. A more detailed view on the temperature evolution of the Doughnut is 


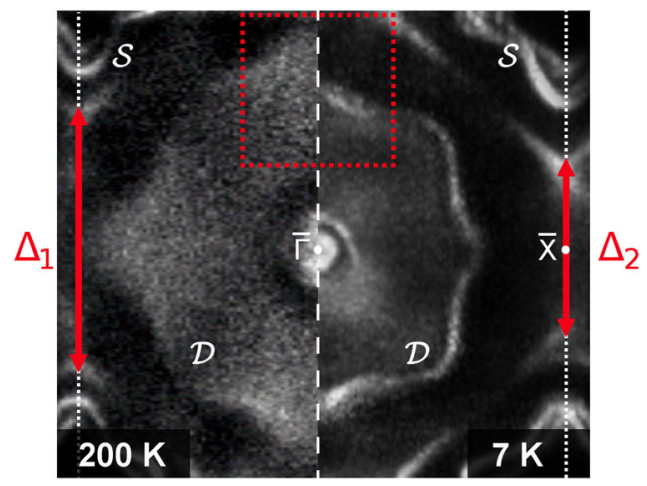

Fig. 2 ARPES view on the bulk-related properties of Eulr ${ }_{2} \mathrm{Si}_{2}$. The temperature-dependent valence change from 2.5 at $200 \mathrm{~K}$ (left) to 2.83 at $7 \mathrm{~K}$ (right) results in a pronounced shrinking of the hole-like Doughnut $\mathcal{D}$ due to increasing band filling. Both maps are taken with photons of $55 \mathrm{eV}$

given in Supplementary Fig. S1. There, we also compare the experimental findings with the results of ab initio density functional theory (DFT) calculations. Moreover, we analyzed the size of the calculated Doughnut for different valences and related it to the experimentally determined temperature dependence of the valence (Supplementary Fig. S2).

The Doughnut is a pure bulk FS sheet and formed by spdderived bands with hole-like dispersion. ${ }^{16,20,21}$ If no $4 f$ states contribute to the $\mathrm{FS}^{20,21}$ the size of $\mathcal{D}$ solely depends on the band filling of the hole-like spd-derived valence band. Since Eulr ${ }_{2} \mathrm{Si}_{2}$ is a mixed-valent compound, the temperature dependence of the Doughnut can be unambiguously understood in terms of gradual filling of the hole-like band with increasing valence, or in other words, decreasing $4 f$ occupancy in favor of $\mathrm{Eu} 5 d$ valence electrons. It follows that the band gets gradually shifted towards higher BEs resulting in a shrinking of the Doughnut. For similar reasons, the size of the $\bar{M}$-gap increases as indicated by the arrows labeled $\Delta_{1}$ and $\Delta_{2}$ in Fig. 2 , which span between the edges of neighboring $\bar{M}$-gaps along the $\bar{M}-\bar{X}-\bar{M}$ direction. Analysis of the results from band structure calculations presented below suggests that a change of the band filling by 0.3 electrons leads to similar modifications of the electronic structure at the Fermi level as seen in ARPES. Thus we conclude that the experimental observations presented above fully confirm the mixed valence of Eulr ${ }_{2} \mathrm{Si}_{2}$ in the bulk in general, and the observed valence changes from XAS in particular. ${ }^{11}$

\section{Spin-orbit coupling and exchange magnetism in SISE}

Now we turn to the main subject of our story by focusing on the surface-related properties of Eulr ${ }_{2} \mathrm{Si}_{2}$ which can be derived from a study of the $\bar{M}$ surface state. In Fig. 3a we show a large segment of the Fermi surface obtained from ARPES at $200 \mathrm{~K}$ together with the results of ab initio band structure calculations in the frame of DFT, presented in Fig. 3b. It grants full access to the subject of interest seen in the first and second SBZ. In the Fermi surface map, one can distinguish the surface-projected bulk states as well as the surface states and resonances. Three most vivid features should be pointed at, which are also typical of $\mathrm{RERh}_{2} \mathrm{Si}_{2}$ materials: (a) the previously discussed Doughnut $\mathcal{D}$, (b) the square-shaped surfaceprojected bulk band gap around the $\bar{M}$-point, and (c) the diamond-shaped $\bar{M}$ surface state $\mathcal{S}$ lying within the $\bar{M}$-gap. The large symmetric splitting of the $\bar{M}$ surface state into an inner and outer Fermi contour (FC) is due to the Rashba effect, ${ }^{22,23}$ which is of considerable strength in presence of the high-atomic-number $5 d$ element Ir.

Comparing the ARPES-derived Fermi surface with the computed one shown in Fig. $3 \mathrm{~b}$ we may conclude that all experimental
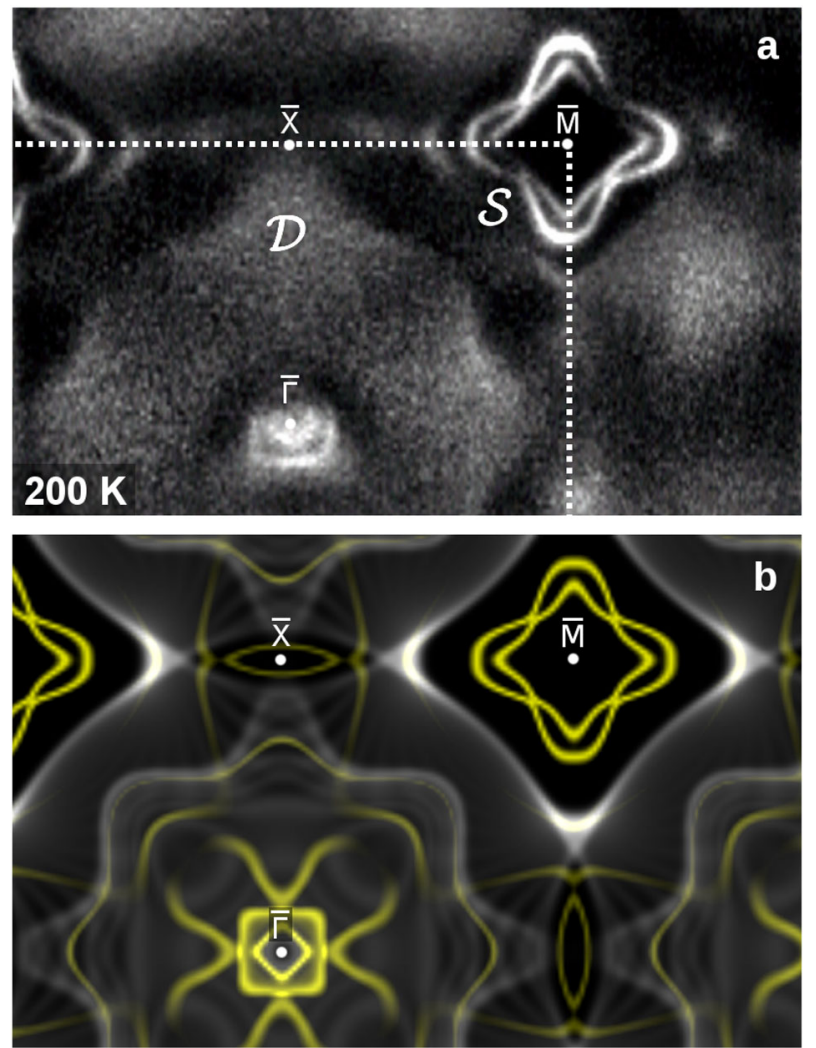

Fig. 3 Fermi surface of Eulr ${ }_{2} \mathrm{Si}_{2}$ for the paramagnetic phase of SISE. a ARPES map taken at $200 \mathrm{~K}$, using photons of $55 \mathrm{eV}$. The Doughnut and the $\bar{M}$ surface state are labeled as $\mathcal{D}$ and $\mathcal{S}$, respectively. In (b) the respective computed Fermi surface is shown as a superposition of projected bulk and slab-derived electron states calculated within DFT. For the bulk the mean $4 f$ occupancy was set to $n_{4 f}=6.5$ which corresponds to the experimentally determined valence $v=2.5$ at $200 \mathrm{~K}^{11}$

findings are nicely reproduced by the ab initio band structure calculations. To simulate the Si-terminated surface, we use a slab geometry where we set the $4 f$ occupancy of the Eu atoms in the fourth, subsurface layer to $\mathrm{n}_{4 f}^{\text {sub }}=7$ and fix the mean occupancy of bulk Eu to $\mathrm{n}_{4 f}^{\text {bulk }}=6.5$. This allows to take into account the divalent subsurface and the mixed-valent bulk of Eulr ${ }_{2} \mathrm{Si}_{2}$. For all Eu atoms we use an unpolarized $4 f$ shell. To emphasize the SISE-related surface electronic structure, we have highlighted the respective bands in yellow while the bulk band states are shown in gray.

As expected for the Rashba effect, the strong spin-orbit coupling (SOC) drives the spins of the surface-state electrons to be parallel to the $a b$-plane and locks them perpendicular to the momentum k. This results in a k-dependent spin polarization accompanied by a symmetric spin-orbit splitting of the $\bar{M}$ surface state. However, our ab initio calculations reveal that the classical Rashba model, ${ }^{22}$ which treats the electron spin and momentum as dynamical variables, is not applicable to Ir-based $\mathrm{REIr}_{2} \mathrm{Si}_{2}$ compounds. ${ }^{24}$ This manifests itself in a complex spin structure, which predetermines the shape of the FCs in the presence of the magnetic order, see below.

Our proposition of divalent Eu in SISE implies that at certain temperatures the Eu $4 f$ moments can be ordered producing a magnetic layer. In that regard the following tasks can be formulated. Experimentally, we need to find, if present at all, the onset of magnetic order of Eu in SISE and map the Fermi surface for this phase. This can be easily done by exploring the evolution of the $\bar{M}$ surface state with temperature, disclosing any possible deviations from its symmetric shape seen in Fig. 3 or changes in 

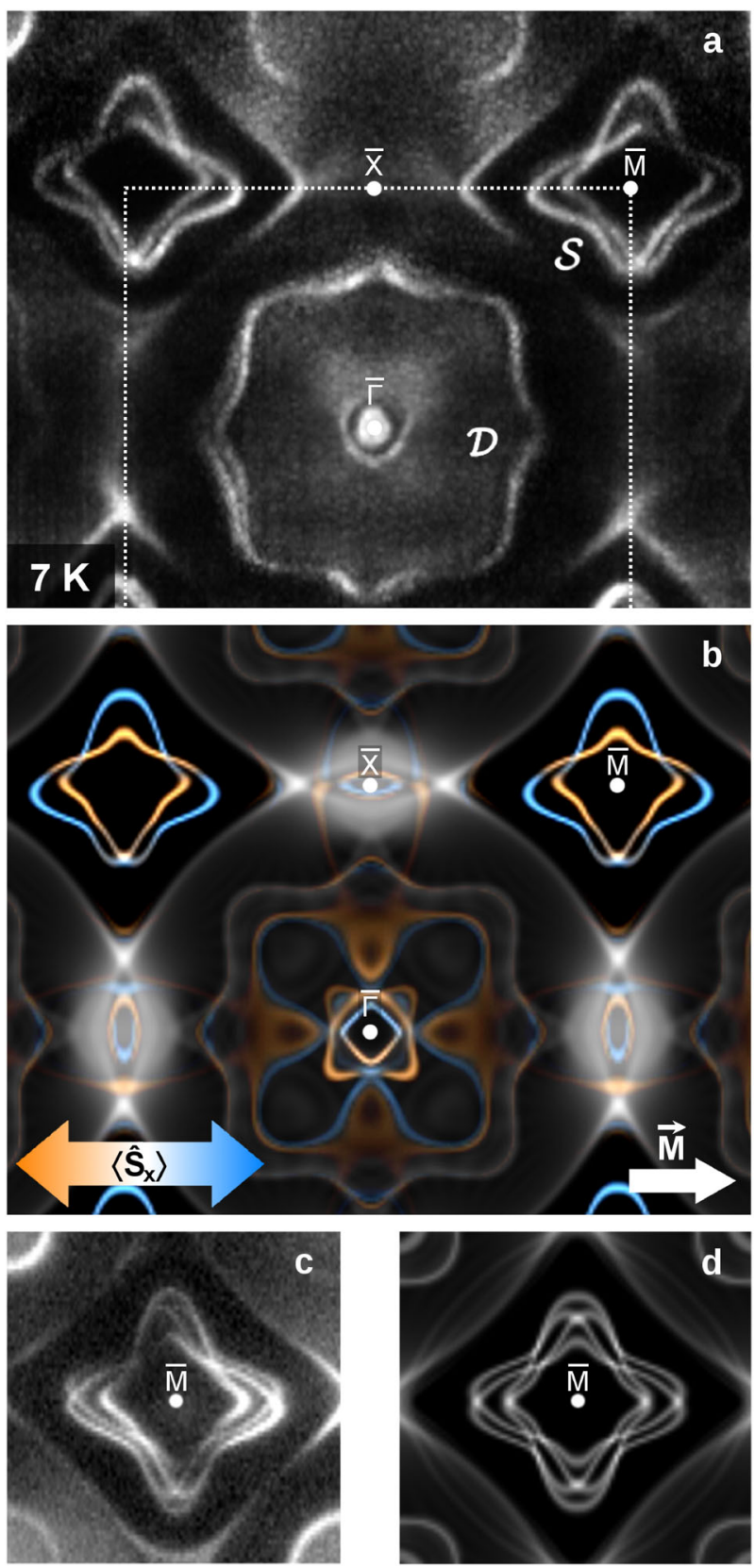

Fig. 4 Fermi surface of Eulr ${ }_{2} \mathrm{Si}_{2}$ for the FM-ordered phase of SISE. a ARPES map taken mainly from a single magnetic domain at $7 \mathrm{~K}$ with photons of $55 \mathrm{eV}$. b Calculated FS shown as a superposition of projected bulk and slab band structure. For the bulk the mean $4 f$ occupancy was set to $n_{4 f}=6.2$ which corresponds to the experimentally determined valence $v=2.83$ at $7 \mathrm{~K}^{11}$. c If two magnetic domains of opposite magnetization are probed simultaneously, ARPES shows the superposition of two diamond-shaped FCs associated with each direction of magnetization. d Two domains simulated by ab initio calculations

the band splitting. Theoretically, we have to compute the Fermi surface in presence of magnetic order in SISE and check to which extent the calculation reproduces the experimental results.

The essential point is how the topology of the $\bar{M}$ surface state may change when the Rashba effect is accompanied by the exchange field of the ordered Eu $4 f$ moments in SISE. From experiments on magnetic metal surfaces, it is well known that a sufficiently large in-plane magnetization affects the Rashba-split surface states, leading to an asymmetric surface-state dispersion for $\mathbf{k}$ perpendicular to the magnetization, ${ }^{25-27}$ which, in turn, modifies the symmetry of the Fermi contours. An out-of plane magnetization does not affect the FC symmetry, but changes the splitting. Therefore, we decided to perform our next experiment at the lowest possible temperature $(7 \mathrm{~K})$, assuming that if the magnetic order sets in at some temperature above, the result of its action on the $\bar{M}$ surface state should be well seen in the ARPESderived Fermi surface. The obtained results are shown in Fig. 4a. Already a brief inspection reveals that there are fundamental changes in the topology of the $\bar{M}$ surface state. We see that the state remains split but now its dispersion reveals strong asymmetry in $\mathbf{k}$-space. This suggests that here the splitting of the state depends not only on SOC but also on the emergent magnetic order of the Eu $4 f s$ in SISE. Then, the appearing exchange-magnetic interaction (EMI) and SOC engage in competition with each other. This results in a strong distortion of the Fermi contours, because EMI favors a collinear alignment of the spins with the magnetization $\mathbf{M}$ and therefore strives to break up the SOC-induced spin-momentum locking. Note here that a strong asymmetry is seen only for the $\bar{M}$ surface state while the bulk Doughnut remains unaffected. It proves that the discussed phenomenon is observed exclusively at the SISE surface-related block of mixed-valent Eulr ${ }_{2} \mathrm{Si}_{2}$.

One can also see that the distortion of the $\bar{M}$ surface state takes place in a well defined direction of the $\mathbf{k}$-space. In the magnetically ordered phase the mutually orthogonal $\bar{M}-\bar{X}$ directions are not equivalent anymore. This point is relevant for DFT calculations where we choose a geometry with the Eu $4 f$ moments aligned ferromagnetically along [100]. The computational results are shown in Fig. $4 \mathrm{~b}$. The spin expectation value $\left\langle\hat{S}_{x}\right\rangle$ along the magnetization direction is shown in light blue and orange color representing spin up and down, respectively. White corresponds to $\left\langle\hat{S}_{x}\right\rangle=0$. To both Fermi contours a dominating spin can be assigned: the outer contour is primarily formed by spin up states, i.e., spins aligned parallel to the magnetization while spin down dominates the inner contour. A closer look, however, reveals that the spin expectation value changes sign upon moving along a single contour in the vicinity of the crossing points emphasizing the strength of SOC in Ir compounds. Apparently, EMI is not strong enough to entirely overcome the SOC induced spin-momentum locking and to align all spins of the $2 \mathrm{D}$ electrons with the magnetization of the Eu $4 f$ moments.

In agreement with the results for other magnetic Rashba systems the calculations show that the direction of the magnetization determines the asymmetric deformation of the Fermi contours, which is always perpendicular to the magnetization and changes sign upon magnetization reversal. A detailed discussion of the evolution of the asymmetry and the spin structure of the surface-state FCs is given below in the frame of an effective $\mathbf{k} \cdot \mathbf{p}$ model.

So far, we discussed the ARPES results obtained from a single magnetic domain. However, it can be that different magnetic domains are probed simultaneously in ARPES measurements. In this case, each magnetic domain will contribute a different distortion to the FS and the resulting ARPES map will comprise a superposition of FS maps from each domain. As an example of such a situation, we present in Fig. $4 c$ a respective ARPES result combined with our simulations for the $\bar{M}$ surface state depicted in Fig. 4d. In order to model two oppositely oriented magnetic domains in a single calculation we use a symmetric, Si-terminated slab in which each of the two surfaces accounts for one magnetic domain. For the computational details we refer to the Theoretical modeling section. As we can see, experiment and theory are in perfect agreement.

Now we turn to the $E(\mathbf{k})$ band maps derived from our ARPES measurements along with the results of ab initio DFT calculations. Between $200 \mathrm{~K}$ down to $\sim 50 \mathrm{~K}$ we did not observe any tangible changes in the spectral pattern reflecting the $\bar{M}$ surface state, 

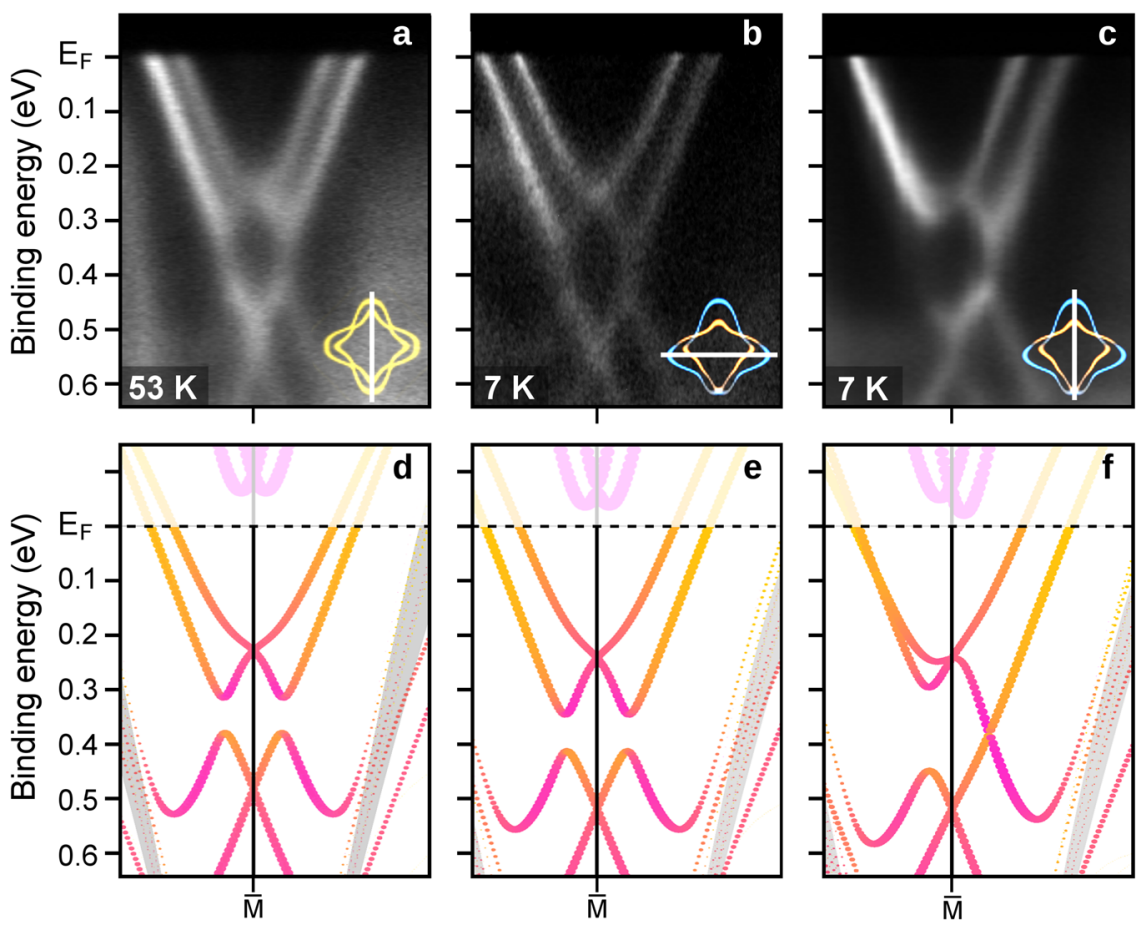

Fig. 5 Modifications of the band dispersion upon entering the FM phase. a-c: ARPES cuts taken at a photon energy $h v=55 \mathrm{eV}$ showing the band dispersion of the $\bar{M}$ surface state in the $\bar{X}-\bar{M}$ direction of the SBZ above $(53 \mathrm{~K})$ and well below (7 K) the ordering temperature of the Eu $4 f$ moments in SISE. In the insets the respective cuts are marked schematically by a white line through the FS. The maps given in (b) and (c) are taken at $7 \mathrm{~K}$ and correspond to mutually orthogonal $\bar{X}-\bar{M}$ paths. $\mathbf{d}-\mathbf{f}$ : The results of respective ab initio band structure calculations. Pink and yellow fat bands highlighting the $\bar{M}$ surface state show the individual weights of $\mathrm{Si}$ in the first and Ir in the second layer of the SISE, respectively. The surface-projected bulk bands are plotted in gray

which retains its fourfold symmetry throughout this temperature range. This can be seen in Fig. $5 \mathrm{a}$, where at $53 \mathrm{~K}$ the $\overline{\mathrm{M}}$ surface state forms a pair of split bands symmetric relative to the $\bar{M}$-point with an electron-like dispersion close to the Fermi level. The respective cut in $\mathbf{k}$-space is shown in the inset. Repeating the ARPES measurement after rotating the sample by $90^{\circ}$ gives a similarly symmetric spectral pattern (not shown here) proving the fourfold symmetry. It is noteworthy that within the examined temperature range there is no detectable gap opening at the $\bar{M}$-point, which is a TRIM (time reversal invariant momentum). This indicates that the split bands are degenerate at the TRIM, as expected for pure spin-orbit splitting. The presence of a magnetization would have been recognized by its characteristic features: an in-plane magnetization causes an asymmetry in the surface-state $E(\mathbf{k})$ and shifts the degeneracy points away from the TRIM, while the out-of plane magnetization lifts the degeneracy. Thus, the observed behavior of the $\bar{M}$ surface state implies that at $53 \mathrm{~K}$ the Eu $4 \mathrm{f}$ moments are not yet ordered and the SISE remains in a paramagnetic state.

In Fig. 5b, c, we show the ARPES maps taken after cooling the sample down to $7 \mathrm{~K}$ before and after rotating the sample by $90^{\circ}$, respectively. In Fig. $5 \mathrm{~b}$ the dispersion seems symmetric with respect to the $\overline{\mathrm{M}}$-point similarly to the $53 \mathrm{~K}$ data, but now the size of the splitting is noticeably larger. However, in the image taken after rotation by $90^{\circ}$ shown in Fig. $5 \mathrm{c}$ the dispersion reveals a strong asymmetry with respect to the $\bar{M}$-point: to the right of $\bar{M}$ we find two branches while to the left the two electron-like bands seem to merge into a single band. Further, the band dispersion near the $\bar{M}$-point contains valuable information on the orientation of the $4 f$ moments with respect to the $c$ axis. Recent studies on $\mathrm{HoRh}_{2} \mathrm{Si}_{2}$ have shown that the degeneracy of the surface state at the $\bar{M}$-point pertinent to the paramagnetic phase is lifted when the moments have a notable out-of-plane component resulting in a Zeeman-like splitting, which can be clearly resolved in the ARPES data. ${ }^{19}$ Since here, within a similar experimental resolution, the degeneracy of the bands remains unlifted in the $7 \mathrm{~K}$ data, we may conclude that the ordered $4 f$ moments of the divalent Eu in SISE remain aligned within the basal plane.

In Fig. 5d-f, we present the calculated band structure underlying the above ARPES band maps. We highlight the $\bar{M}$ surface state by fat bands that show the joint contribution of the topmost $\mathrm{Si}$ - and adjacent Ir-atomic layer. Note that the experimentally observed non-dispersive bands representing the $4 f^{6}$ final state multiplet are absent in the computed band structure. This is because the $4 f$ electrons were treated within the open-core approach (see the Theoretical modeling section). Figure $5 \mathrm{~d}$ accounts for the paramagnetic configuration of the subsurface of Eulr ${ }_{2} \mathrm{Si}_{2}$, while in Fig. $5 \mathrm{e}$, $\mathrm{f}$ the calculated bands for the magnetic phase are shown along the horizontal and vertical $\bar{X}-\bar{M}$ path, respectively. As seen in the figures, our calculations correctly describe the dispersion of the $\bar{M}$ surface state in the paramagnetic phase, where the band splitting arises solely due to SOC. In the magnetic phase, by aligning the subsurface $4 f$ moments ferromagnetically along [100], we also highly accurately reproduce the experimentally observed changes, when the emerging EMI competes with SOC. From our calculations, we can also extract information about the fine details of the surface state dispersion, which cannot be experimentally resolved. For example, the seeming merger into a single band, Fig. 5c, in fact corresponds to two branches of a split band that become substantially less separated in energy and cross at a point in the $\bar{X}-\bar{M}$ line, Fig. $5 f$. The in-plane magnetization causes an insignificant shift of the degeneracy points, giving rise to a tiny gap (less than $1 \mathrm{meV}$ ) at $\overline{\mathrm{M}}$. Above the Fermi level one can see an unoccupied surface state; it is localized at the topmost Si layer and is affected by the magnetization in a manner similar to the Rashba-split surface state on magnetic metal surfaces. 

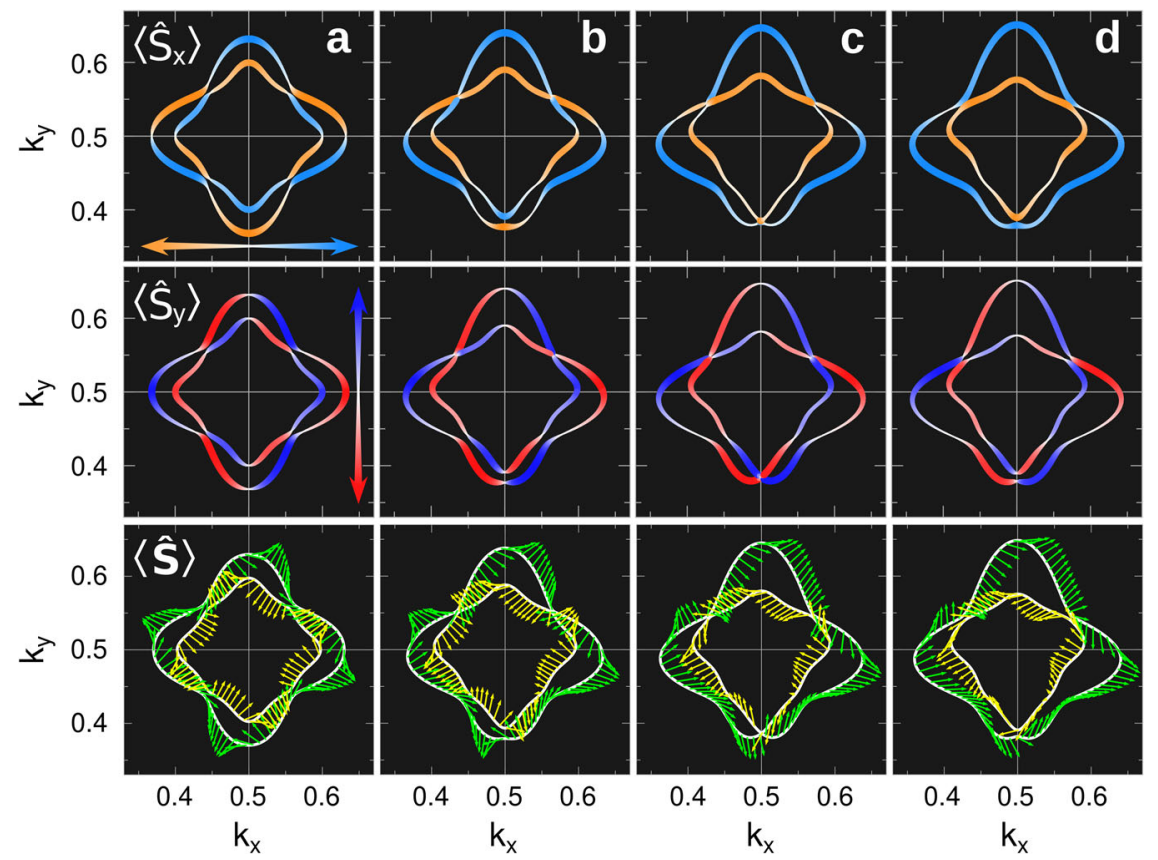

Fig. 6 Spin-resolved Fermi contours for the $\bar{M}$ surface state. The contours for the surface state of Eulr ${ }_{2} \mathrm{Si}_{2}$ by the effective $\mathbf{k} \cdot \mathbf{p}$ model around $\bar{M}$ [see Supplementary Fig. S6(b)] are shown for the paramagnetic phase (a) and for three model magnetic phases characterized by the exchange-interaction parameter $\mathcal{J}=50 \mathrm{meV}(\mathbf{b}), \mathcal{J}=90 \mathrm{meV}(\mathbf{c})$, and $\mathcal{J}=115 \mathrm{meV}$ (d). For each phase, the expectation values of $\left\langle\hat{S}_{x}\right\rangle$ (top row) and $\left\langle\hat{S}_{y}\right\rangle$ (middle row) are presented. The resulting in-plane orientation of the spins, $\left\langle\widehat{\mathbf{S}}_{\rangle}=\left\langle\hat{S}_{x}\right\rangle \widehat{\mathbf{x}}+\left\langle\hat{S}_{y}\right\rangle \widehat{\mathbf{y}}\right.$, is indicated by yellow (green) arrows for the inner (outer) contour in the bottom row. In the magnetic phases, the $4 f$ moments of the subsurface Eu atomic layer are implied to be ferromagnetically ordered along the $x$ axis

In our next experiment, we studied the temperature scale for the onset of magnetism in SISE. To this end, we explored the spectral pattern reflecting the $\bar{M}$ surface state in dependence on temperature, starting from $7 \mathrm{~K}$ and following the modification of the asymmetric band dispersion. The obtained results are shown in the Supplementary Materials (Supplementary Fig. S3) where also a detailed discussion of the experimental results is given (Supplementary Note 2). We found that magnetic order in SISE sets in at about $48 \mathrm{~K}$, which is close to the respective FM surface ordering temperature of $42 \mathrm{~K}$ observed for the layered antiferromagnet $\mathrm{EuRh}_{2} \mathrm{Si}_{2}$. ${ }^{19}$

The ferromagnetic order in the SISE block concluded from our ARPES measurements is independently confirmed also by magnetic circular and linear dichroism measurements which we performed at the Eu $M_{4,5}$ absorption edges at low temperature. As shown in Supplementary Note 3, we observe X-ray magnetic circular dichroism (XMCD) and X-ray magnetic linear dichroism (XMLD) signatures in the absence of an external magnetic field. The detected dichroic signals can only be explained by in-plane ferromagnetic order in the SISE block with moments aligned along the (100) direction, which is in full agreement with what has been observed in ARPES.

\section{Competing effect of two spin-splitting mechanisms}

To gain further insight into the peculiarities of the competition between SOC and EMI and to follow the modification of the surface-state Fermi contours by EMI under gradually increasing its strength we use the effective $\mathbf{k} \cdot \mathbf{p}$ model developed in ref. ${ }^{24}$ based on the methodology of ref. ${ }^{28}$. The model employs a fully ab initio derived relativistic $\mathbf{k} \cdot \mathbf{p}$ Hamiltonian and is thereby capable of accurately reproducing the spin polarization of the spin-orbit split surface states. To simulate the effect of the magnetic exchange interaction, we include into the model the exchange term originating from the scalar product $-J_{\mathrm{ex}} \mathbf{M} \cdot \boldsymbol{\sigma}$, see, e.g., ref. ${ }^{29}$ ( $\boldsymbol{\sigma}$ is the vector of the Pauli matrices). Thus, the tunable parameter of the model is $\mathcal{J} \equiv J_{\mathrm{ex}} \mathbf{M}$, the magnetization $\mathbf{M}$ scaled by the strength of exchange interaction $J_{\text {ex }}$ (see Supplementary Note 4).

We start with the paramagnetic phase of $\mathrm{Eulr}_{2} \mathrm{Si}_{2}, \mathcal{J}=0$. The spin-resolved contours obtained within the $\mathbf{k} \cdot \mathbf{p}$ model are shown in Fig. 6a [cf. the LDA Fermi contours in Supplementary Fig. S6(c)]: the sign and the absolute value of the spin $x$ - and $y$-projections are seen to vary consistently along the outer and inner contour, reflecting a SOC induced spin-momentum locking around the point $\bar{M}$, which has the same symmetry as $\bar{\Gamma}$. Due to the symmetry, for example, the $\left\langle\hat{S}_{x}\right\rangle$ contours are the $\left\langle\hat{S}_{y}\right\rangle$ ones rotated clockwise by $\pi / 2$. These symmetric Fermi contours with the specific spinmomentum locking different from the classical Rashba splitting will serve as a reference state in our further analysis of the competing effect of SOC and EMI on the behavior of the surface state. We thereby introduce the exchange interaction in the presence of the Rashba effect. Note that the spin structure of the surface state shown in the bottom row of Fig. $6 a$ is characterized by a triple spin winding around the contour. One could heuristically reduce it to a k-cubic model containing the term $\left(k_{-}^{3} \sigma_{-}-k_{+}^{3} \sigma_{+}\right)$in the style of the Rashba or Dresselhaus twoband Hamiltonian for $m_{j}= \pm 3 / 2$ states of semiconductor quantum wells. ${ }^{30-32}$ However, although at the $\bar{M}$-point this surface state is of predominantly Ir $d_{x^{2}-y^{2}}$ character, ${ }^{24}$ it has neither Rashba nor Dresselhaus spin structure, thus complementing these two wellknown spin-orbit-induced patterns.

In the magnetic phase of Eulr ${ }_{2} \mathrm{Si}_{2}$, the in-plane orientation (as before, along the $x$ axis, i.e., $\mathcal{J}=\mathcal{J} \hat{\mathbf{x}}$ of the ferromagnetically ordered $4 f$ moments in the subsurface rare-earth layer is implied. By continuously increasing the parameter $\mathcal{J}$ accounting for EMI, we can visualize how the setting up of the magnetic order modifies the spin-structure and the shape of the Fermi contours. As seen in Fig. $6 \mathrm{~b}$, an increase of the $\mathcal{J}$ value up to $50 \mathrm{meV}$ is sufficient to cause the marked asymmetry: We mainly observe an asymmetric stretch-out of the contours (in opposite directions for the outer and the inner one) along the $y$ axis-the line 
perpendicular to the magnetization direction. Keeping in mind the form of the exchange term, a simple picture of the deformation is that the 'orange' (light blue) states tend to decrease (increase) their binding energy and thus move towards (away from) the $\bar{M}$-point the stronger the more intense is the color, i.e., the absolute value of $\left\langle\hat{S}_{x}\right\rangle$. As regards to the resulting spin structure, it has not undergone any tangible changes: it mostly preserves the spin-momentum locking similar to that in the paramagnetic state, Fig. $6 \mathrm{~b}$.

Next, we reach the $\mathcal{J}$ value of 90 meV that models the Eu $4 f$ moments of the magnetic Eulr ${ }_{2} \mathrm{Si}_{2}$, Fig. $6 \mathrm{c}$. For this value of the exchange-interaction parameter, the shape of the Fermi contours and the detailed behavior of the spin $x$-projection by the $\mathbf{k} \cdot \mathbf{p}$ model are in full accord with the ab initio calculations shown in Fig. 4b. Here, the $\left\langle\hat{S}_{x}\right\rangle$ component almost completely loses its SOCinduced variations along the contours except for rather small fragments between $k_{y}=0.5$ and $0.552 \pi / a$, while the $\left\langle\hat{S}_{y}\right\rangle$ spin structure with the specific spin-momentum locking is mainly preserved. This means that perhaps a stronger $4 f$ magnetism is necessary to 'decouple' spin from momentum. Actually, a further increase of $\mathcal{J}$ by $\sim 30 \%$ (up to $115 \mathrm{meV}$ ) leads to a spin structure in which $\left\langle\hat{S}_{x}\right\rangle$ has the same sign everywhere in the contour, Fig. $6 \mathrm{~d}$. In this case, EMI dominates over SOC. However, the specific pattern of the reduced $\left\langle\hat{S}_{y}\right\rangle$ component along the contours still resembles the original spin-momentum locking. In the bottom row of Fig. 6, we visualize the overall effect of the emerging exchange interaction on the constant energy curves CECs and the spin orientation, as described within our effective $\mathbf{k} \cdot \mathbf{p}$ model with increasing the exchange parameter $\mathcal{J}$. Thereby, we clearly show how the spin polarization of the surface-state electrons can be gradually tuned, producing a specific spin structure, which, to our best knowledge, has not been reported before.

We wish to note that the polarized state corresponding to Eu $4 f$ moments oriented along the $c$ axis cannot be realized in an ARPES experiment, but it can likely easily be realized outside of an ARPES spectrometer by applying a small external magnetic field. The magnetic anisotropy of divalent Eu systems is usually very weak because the magnetic moment of divalent $\mathrm{Eu}$ is a pure $\mathrm{S}$ state and therefore does not lead to a first order crystal electric field effect as in most other rare earths. Therefore a tiny external field is expected to be able to rotate the Eu moments in any direction in the basal plane or along the $c$ axis. Thus using a small external field it should be easy to produce a very large single polarization domain, to switch from an $x$-oriented domain to a $y$-oriented domain, or to induce the c-oriented domain. This opens the way for a highly tunable polarized surface state. Moreover, the temperature-dependent mixed-valence property of the bulk also allows to tune the energy and momentum size of the projected bulk band gaps to which the 2D carriers are confined. This gives an additional degree of freedom to handle the spin-polarized carriers at the surface of an overall non-magnetic crystal.

In summary, we provide clear evidence for strong twodimensional ferromagnetism at the surface of $\mathrm{Eulr}_{2} \mathrm{Si}_{2}$ despite this compound is non-magnetic in the bulk due to a mixed-valent Eu state. This surface magnetism appears because the relaxation of the top Si-Ir-Si trilayer switches the underlying Eu layer from the non-magnetic valence fluctuating state to the magnetic divalent state. This Eu layer strongly couples magnetically to 2D electrons in a surface state located in the Si-Ir-Si trilayer. These 2D electrons are also subject to a strong Rashba effect due to the large spinorbit coupling of Ir and the absence of inversion symmetry at the surface. The beauty of this system lies in the temperature dependent ordering of the Eu $4 f$ magnetic moments in the subsurface layer, which results in an impressive change in the spin polarization of the $2 \mathrm{D}$ electrons. At high temperatures the $4 \mathrm{fs}$ are disordered. Nevertheless the 2D electrons present a strong momentum dependent in-plane spin polarization due to the Rashba effect. But below $48 \mathrm{~K}$ the magnetic moments of Eu in the subsurface layer become ferromagnetically ordered with a large moment pointing along one of the basal plane crystallographic axes. The resulting strong magnetic exchange competes with the spin-orbit coupling and changes the spin polarization of the 2D electrons in a remarkable way. The detailed insight into the changes of the spin polarization by interplay between spin-orbit coupling and exchange magnetic interaction is gained by DFT calculations and a DFT-based effective $\mathbf{k} \cdot \mathbf{p}$ model, which nicely reproduce the experimental results. This provides a sound basis for a systematic search for further appropriate materials. Furthermore the 2D electrons and its spin polarization can be manipulated by applying an external magnetic field or tuning the valence of the Eu in the bulk, e.g., using chemical substitution.

Finally, our results call for the reconsideration of a large class of materials containing heavy $5 d$ elements together with rare-earth or transition metal elements, which show mixed-valent or paramagnetic bulk properties. Just like $\mathrm{Eulr}_{2} \mathrm{Si}_{2}$, many of them may exhibit unique magnetic properties at the surface which have been overlooked so far.

\section{METHODS}

Experimental details

The temperature-dependent ARPES experiments from $200 \mathrm{~K}$ down to $7 \mathrm{~K}$ were performed at the 105 beamline of the Diamond Light Source (DLS). ${ }^{33}$ High quality single crystalline samples of Eulr $\mathrm{Si}_{2}{ }^{11}$ were cleaved in-situ under ultra-high vacuum conditions.

XMCD and XMLD measurements were performed at beamline ID32 of the European Synchrotron Radiation Facility (ESRF).

\section{Theoretical modeling}

$\mathrm{Ab}$ initio calculations were performed in the frame of Density Functional Theory (DFT) using the Full-Potential non orthogonal Local-Orbital minimum-basis band-structure scheme (FPLO) described in ref. ${ }^{34}$ in the Local Spin Density Approximation (LSDA). The bulk crystal we modeled in space group (SG) $14 / \mathrm{mmm}$ taking maximal advantage of symmetry in the $\mathrm{ThCr}_{2} \mathrm{Si}_{2}$ family. Lattice parameters and the $z$ position of the $\mathrm{Si}$ atom (Wyckoff position $4 e$ ) were optimized with respect to the total energy. To model the surface we use a slab geometry. A Si-terminated symmetric slab consisting of 19 atomic layers and an asymmetric slab of 32 atomic layers with $\mathrm{Si}$ termination on one and Eu termination on the other side were modeled in SGs $P 4 / \mathrm{nmm}$ and $P 4 \mathrm{~mm}$, respectively. Relaxation of the surface was taken into account for the four utmost layers.

In all calculations the Eu $4 f$ electrons were treated within the so-called open-core approximation, i.e., the Eu $4 f$ states were removed from the valence basis and entered the theory as core orbitals. In doing so, the mean $4 f$ occupancy $n_{4 f}$ is fixed to a given value, which, in our calculations, was the experimentally determined valence. To simulate our ARPES data taken at $7 \mathrm{~K}(200 \mathrm{~K})$ we derived the corresponding bulk occupation number $\mathrm{n}_{4 f}=6.2(6.5)$ from the experimental value $v=2.8(2.5)$ of the valence determined by Mössbauer ${ }^{9}$ and XAS ${ }^{11}$ measurements. Assuming that $\mathrm{Eu}$ is divalent at the surface and in the subsurface the associated occupancy was fixed to $\mathrm{n}_{4 f}=7$, while the remaining layers where treated as in the bulk. To model the paramagnetic phase we use an unpolarized $4 f$ shell. For simulation of the low temperature phase all Eu $4 f$ electrons at the surface in the fourth layer were assumed to have parallel spins with the resulting moments aligned along the [100] direction.

To simulate our ARPES results for two magnetic domains [see Fig. 4c] we perform a single calculation using the Si terminated symmetric slab of 19 atomic layers described above with the Eu $4 f$ moments in the subsurface layer aligned along [100]. Thereby we exploit the fact that in the paramagnetic phase the spatial inversion symmetry of the slab leads to twofold degenerate surface states with the hidden spin polarization ${ }^{35}$ opposite at the two surfaces of the slab. This means that the same direction of the in-plane magnetization at both the two surfaces results in the distortion of the surface state bands in opposite directions. The same result can be achieved by two calculations in which only one of the surfaces is considered with opposite alignment of the $4 f$ moments, respectively (see also ref. ${ }^{26}$ ).

The ab initio calculations underlying the $\mathbf{k} \cdot \mathbf{p}$ model were performed for the Si-terminated (001) surface of the paramagnetic Eulr ${ }_{2} \mathrm{Si}_{2}$ with the extended linear augmented plane wave method ${ }^{36}$ (the accuracy of the 
wavefunctions is essential for the efficiency of the $\mathbf{k} \cdot \mathbf{p}$ method) within the local density approximation (LDA) for the exchange-correlation functional using the full-potential scheme of ref. ${ }^{37}$ (see Supplementary Note 4 for details of the $\mathbf{k} \cdot \mathbf{p}$ model and LDA calculations).

\section{DATA AVAILABILITY}

All relevant data are available from the corresponding author upon request.

\section{ACKNOWLEDGEMENTS}

This work was supported by the German Research Foundation (DFG) through Grants No. KR3831/5-1, No. LA655/20-1 and Fermi-NEst. Support was provided by the Spanish Ministry of Science, Innovation and Universities (Project No. FIS2016-76617-P and No. FIS2016-75862-P). We acknowledge financial support from the Spanish Ministry of Economy (MAT-2017-88374-P). The authors also acknowledge support by Saint Petersburg State University (Grant No. 15.61.202.2015) and Tomsk State University Academic D. I. Mendeleev Fund Program (Research Grant No. 8.1.01.2018). We acknowledge Diamond Light Source for access to beamline 105 (proposals no. SI18844-1 and SI17761-1) that contributed to the results presented here.

\section{AUTHOR CONTRIBUTIONS}

C.K. and D.V.V. designed the research; S.Se. and C.G. grew and characterized the samples; S.Sc., M.G., G.P., A.G., S.D., A.C., and D.V.V. performed the UV-ARPES experiments; K.K. performed and analyzed the XMCD and XMLD measurements; theoretical studies were performed by S.SC., I.A.N. and E.E.K.; operation of the UVARPES facility was carried out by T.K.K. and P.D.; the obtained results were discussed together with E.V.C., A.Yu.V., C.L., K.KI., K.K., and C.K.; The manuscript was written by S. Sc., I.A.N., E.E.K., and D.V.V.

\section{ADDITIONAL INFORMATION}

Supplementary Information accompanies the paper on the npj Quantum Materials website (https://doi.org/10.1038/s41535-019-0166-z).

Competing interests: The authors declare no competing interests.

Publisher's note: Springer Nature remains neutral with regard to jurisdictional claims in published maps and institutional affiliations.

\section{REFERENCES}

1. Stewart, G. R. Non-Fermi-liquid behavior in $d$ and $f$-electron metals. Rev. Mod. Phys. 83, 797-855 (2001).

2. Lawrence, J. M., Riseborough, P. S. \& Parks, R. D. Valence fluctuation phenomena. Rep. Prog. Phys. 44, 1-84 (1981).

3. Riseborough, P. S. \& Lawrence, J. M. Mixed valent metals. Rep. Prog. Phys. 79, 084501 (2016)

4. Melsen, J., Wills, J. M., Johansson, B. \& Eriksson, O. Calculations of valence stabilities for the lanthanide metals. J. Alloy. Compd. 209, 15-24 (1994).

5. Fournes, L., Chevalier, B., Lloret, B. \& Etourneau, J. Valence change of europium in the $\mathrm{Eu}\left(\mathrm{Ir}_{(1-x)} \mathrm{Pd}_{x}\right)_{2} \mathrm{Si}_{2}$ silicides. Z. Phys. B Condens. Matter 75, 501-505 (1989).

6. Röhler, J., Wohlleben, D., Kaindl, G. \& Balster, H. Energy balance of mixed-valent Eu ions. Phys. Rev. Lett. 49, 65-68 (1982).

7. Sampathkumaran, E. V. et al. A new and unique Eu-based mixed-valence system - $\mathrm{EuPd}_{2} \mathrm{Si}_{2}$. J. Phys. C: Solid State Phys. 14, L237-L241 (1981).

8. Nagarajan, R. et al. Mössbauer and X-ray absorption spectroscopy on the new mixed-valence system EuNi ${ }_{2} \mathrm{P}_{2}$. Phys. Lett. A 84, 275-277 (1981).

9. Chevalier, B., Coey, J. M. D., Lloret, B. \& Etourneau, J. Eulr ${ }_{2} \mathrm{Si}_{2}$ - a new intermediate mixed valence compound. J. Phys. C. Solid State Phys. 19, 4521-4528 (1986).

10. Seiro, S. \& Geibel, C. From stable divalent to valence-fluctuating behavior in Eu $\left(\mathrm{Rh}_{1-x} \mid \mathrm{r}_{x}\right)_{2} \mathrm{Si}_{2}$ single crystals. J. Phys. Condens. Matter 23, 375601 (2011).

11. Seiro, $\mathrm{S}$. et al. Charge, lattice and magnetism across the valence crossover in Eulr ${ }_{2} \mathrm{Si}_{2}$ single crystals. J. Phys. Condens. Matter. https://doi.org/10.1088/1361648X/ab1509 (2019).

12. Höppner, M. et al. Interplay of Dirac fermions and heavy quasiparticles in solids. Nat. Commun. 4, 3171 (2013).

13. Martensson, $\mathrm{N}$. et al. Highly resolved surface shifts in a mixed-valent system: $\mathrm{EuPd}_{2} \mathrm{Si}_{2}$. Phys. Rev. B 25, 1446-1448 (1982).
14. Mimura, K. et al. Bulk-sensitive high-resolution photoemission study of a temperature-induced valence transition system $\mathrm{EuPd}_{2} \mathrm{Si}_{2}$. J. Electron Spectrosc. Relat. Phenom. 137, 529-533 (2004).

15. Kimura, $\mathrm{S}$. et al. Temperature-induced valence transition of $\operatorname{EuNi}_{2}\left(\mathrm{Si}_{0.25} \mathrm{Ge}_{0.75}\right)_{2}$ studied by Eu $4 d \rightarrow 4 f$ resonant photoemission and optical conductivity. J. Phys. Soc. Jpn. 71, 255-257 (2002).

16. Güttler, M. et al. Tracing the localization of $4 f$ electrons: Angle-resolved photoemission on $\mathrm{YbCO}_{2} \mathrm{Si}_{2}$, the stable trivalent counterpart of the heavy-fermion $\mathrm{YbRh}_{2} \mathrm{Si}_{2}$. Phys. Rev. B 90, 195138 (2014).

17. Chikina, A. et al. Strong ferromagnetism at the surface of an antiferromagnet caused by buried magnetic moments. Nat. Commun. 5, 3171 (2014).

18. Güttler, M. Robust and tunable itinerant ferromagnetism at the silicon surface of the antiferromagnet $\mathrm{GdRh}_{2} \mathrm{Si}_{2}$. Sci. Rep. 6, 24254 (2016).

19. Generalov, A. et al. Spin orientation of two-dimensional electrons driven by temperature-tunable competition of spin-orbit and exchange-magnetic interactions. Nano Lett. 17, 811-820 (2017).

20. Kummer, K. et al. Temperature invariant Fermi surface in the Kondo lattice $\mathrm{YbRh}_{2} \mathrm{Si}_{2}$. Phys. Rev. X 5, 011028 (2015).

21. Rourke, P. M. C. et al. Magnetic-field dependence of the $\mathrm{YbRh}_{2} \mathrm{Si}_{2}$ Fermi surface. Phys. Rev. Lett. 101, 237205 (2008).

22. Bychkov, Yu. A. \& Rashba, E. I. Properties of a 2D electron gas with lifted spectral degeneracy. JETP Lett. 39, 78-81 (1984).

23. Generalov, A. et al. Strong spin-orbit coupling in the noncentrosymmetric Kondo lattice. Phys. Rev. B 98, 115157 (2018).

24. Nechaev, I. A. \& Krasovskii, E. E. Relativistic splitting of surface states at Siterminated surfaces of the layered intermetallic compounds $R_{2} \mathrm{Si}_{2}(\mathrm{R}=$ rare earth, $\mathrm{T}=\mathrm{Ir}$, Rh). Phys. Rev. B 98, 245415 (2018).

25. Krupin, O. et al. Rashba effect at magnetic metal surfaces. Phys. Rev. B 71, 201403 (2005).

26. Krupin, O. et al. Rashba effect at the surfaces of rare-earth metals and their monoxides. New J. Phys. 11, 013035 (2009).

27. Carbone, C. et al. Asymmetric band gaps in a Rashba film system. Phys. Rev. B 93, 125409 (2016).

28. Nechaev, I. A. \& Krasovskii, E. E. Relativistic k - p Hamiltonians for centrosymmetric topological insulators from ab initio wave functions. Phys. Rev. B 94, 201410 (2016).

29. Manchon, A. \& Zhang, S. Theory of spin torque due to spin-orbit coupling. Phys. Rev. B 79, 094422 (2009).

30. Winkler, R Spin-Orbit Coupling Effects in Two-Dimensional Electron and Hole Systems. (Springer: Berlin, 2003).

31. Gerchikov, L. G. \& Subashiev, A. V. Spin splitting of size-quantization subbands in asymmetric heterostructures, Soviet physics. Semiconductors 26, 73 (1992).

32. Iordanskii, S. V., Lyanda-Geller, Yu. B. \& Pikus, G. E. Weak localization in quantum wells with spin-orbit interaction. JETP Lett. 60, 206 (1994).

33. Hoesch, M. A facility for the analysis of the electronic structures of solids and their surfaces by synchrotron radiation photoelectron spectroscopy. Rev. Sci. Instrum. 88, 013106 (2017).

34. Koepernik, K. \& Eschrig, H. Full-potential nonorthogonal local-orbital minimumbasis band-structure scheme. Phys. Rev. B 59, 1743-1757 (1999).

35. Zhang, X., Liu, Q., Luo, J.-W., Freeman, A. J. \& Zunger, A. Hidden spin polarization in inversion-symmetric bulk crystals. Nat. Phys. 10, 387 (2014).

36. Krasovskii, E. E. Accuracy and convergence properties of the extended linear augmented-plane-wave method. Phys. Rev. B 56, 12866-12873 (1997).

37. Krasovskii, E. E., Starrost, F. \& Schattke, W. Augmented Fourier components method for constructing the crystal potential in self-consistent band-structure calculations. Phys. Rev. B 59, 10504-10511 (1999).

Open Access This article is licensed under a Creative Commons Attribution 4.0 International License, which permits use, sharing, adaptation, distribution and reproduction in any medium or format, as long as you give appropriate credit to the original author(s) and the source, provide a link to the Creative Commons license, and indicate if changes were made. The images or other third party material in this article are included in the article's Creative Commons license, unless indicated otherwise in a credit line to the material. If material is not included in the article's Creative Commons license and your intended use is not permitted by statutory regulation or exceeds the permitted use, you will need to obtain permission directly from the copyright holder. To view a copy of this license, visit http://creativecommons. org/licenses/by/4.0/.

(c) The Author(s) 2019 